\title{
New light on the mechanism of phototransduction in phototropin.
}

L. Henry, ${ }^{\ddagger}$ O. Berntsson, ${ }^{\ddagger} \|$ M. R. Panman, ${ }^{\ddagger}$ A. Cellini, ${ }^{\ddagger}$ A. J. Hughes, ${ }^{\ddagger}$ I. Kosheleva, ${ }^{\S}$ R. Henning, ${ }^{\S}$ and S. Westenhoff ${ }^{\ddagger}$

${ }^{\ddagger}$ Department of Chemistry and Molecular Biology, University of Gothenburg, 40530 Gothenburg,Sweden ${ }^{\S}$ Center for Advanced Radiation Sources, The University of Chicago, Chicago IL 60637, USA

"MAX IV Laboratory, Lund University, P.O. Box 118 Lund 221 00, Sweden

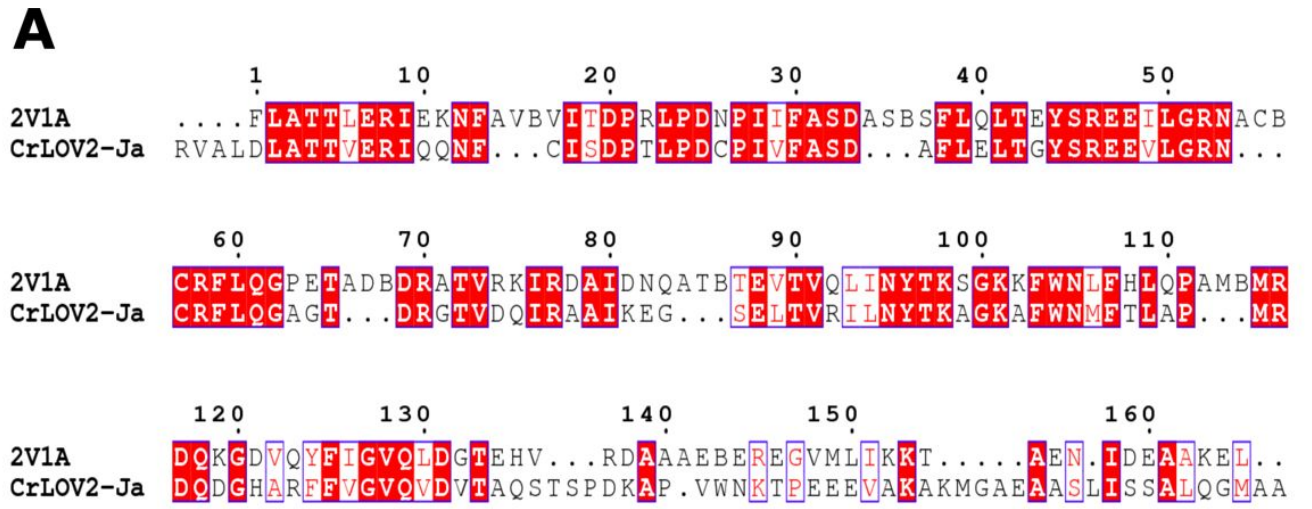

2V1A

CrLOV2-Ja

B

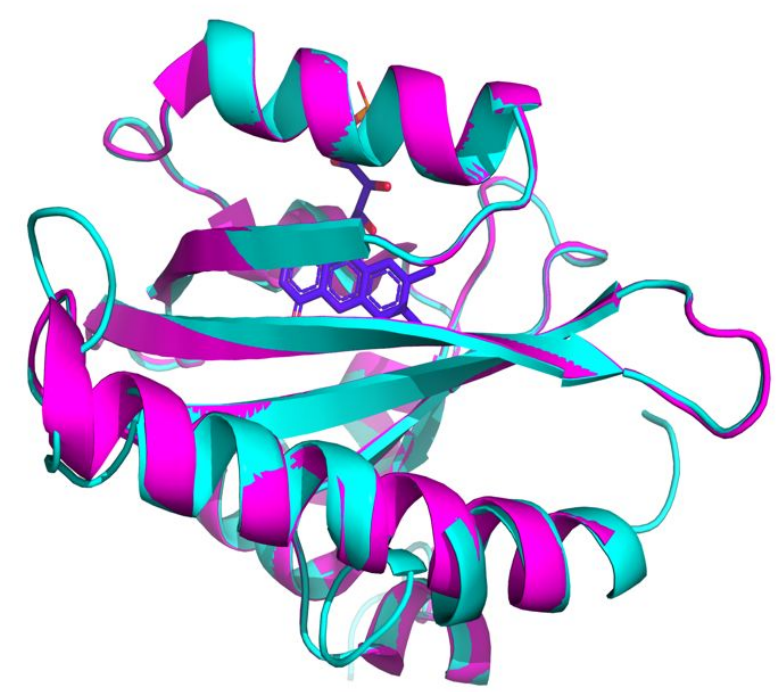

Figure S1: Sequence alignment and homology model of CrLOV2-J $\alpha$ against AsLOV2-Ja.

(A) AsLOV2-J $\alpha$ (PDB: 2V1A) and CrLOV2-J $\alpha$ sequence (NCBI entry: XP_001693387.1; residues: 199-360) were aligned in $\mathrm{T}^{-\mathrm{Coffee}^{1}}$ and visualized in ESPript ${ }^{2}$ (freely accessible online). The T-coffee alignment shows an identity and a similarity of $44.2 \%$ between the two sequences. (B) Superimposition of AsLOV2-Ja (PDB: 2V1A; magenta) and the homology model of CrLOV2-J $\alpha$ (light blue). AsLOV2-J $\alpha$ structure and the alignment in section A were used to build the homology model of CrLOV2-J $\alpha$ in MODELLER ${ }^{3}$. The computed model GA341 score value is 1 whereas its ZDOPE score is equal to -0.72 . 


\section{Supplementary Material}
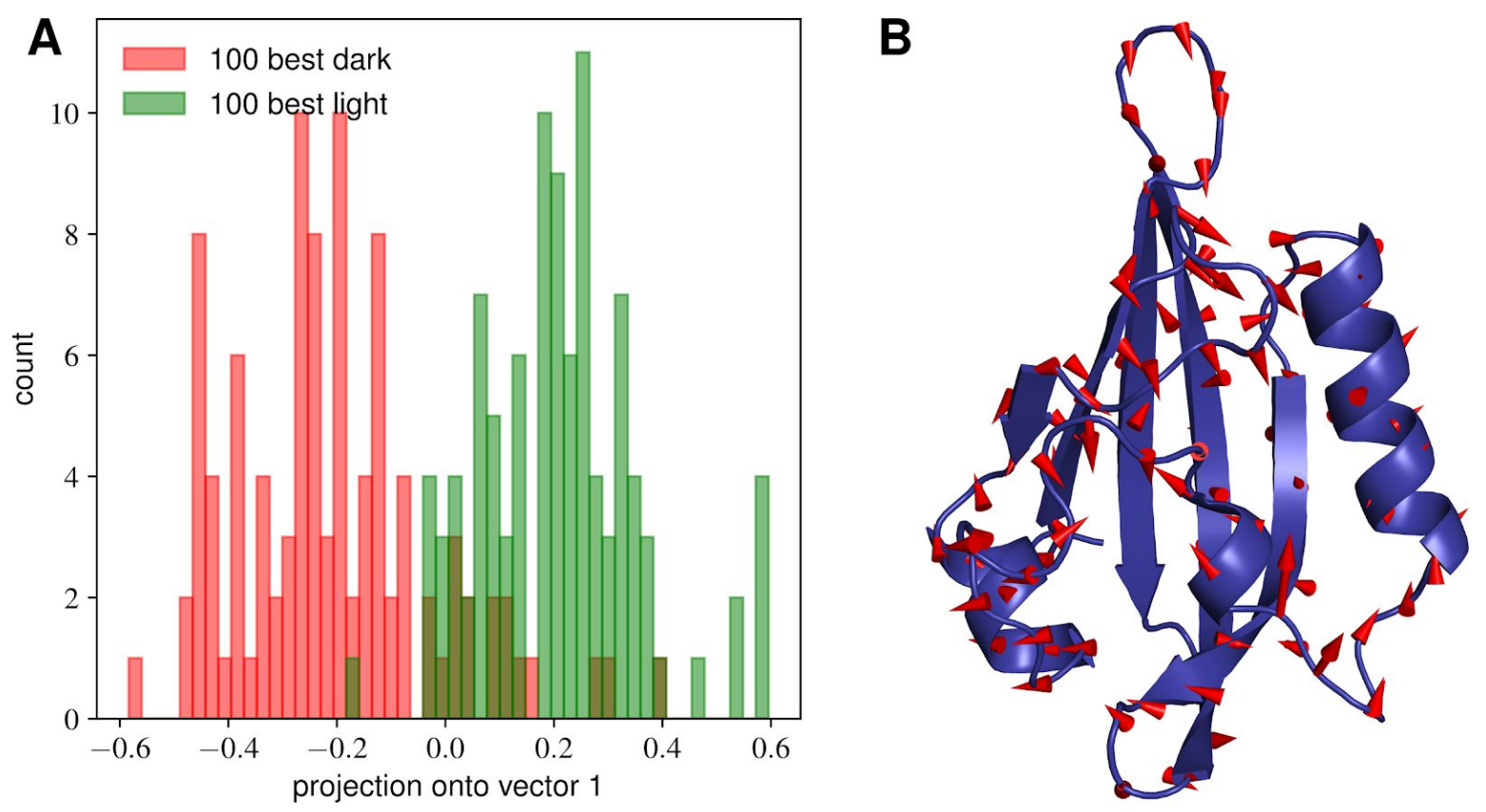

Figure S2: A Histogram of the projections of the 100 best light and 100 best dark structures on the first eigenvector of a PCA. B the primary motion of the $\mathrm{C} \alpha$ atoms on the dark state structure (PDB: 1N9L) along the first eigenvector of the PCA. The length of the arrows are doubled to accentuate the motion. 
Table S1: List of the hydrogen bonds present in more than $10 \%$ of the dark and light best models. Hydrogen bonds with an occupancy deviation superior to $50 \%$ between the dark and the light states, relative to the dark state, are highlighted in bold.

\begin{tabular}{|c|c|c|c|c|}
\hline \multirow{2}{*}{\multicolumn{2}{|c|}{ Donor }} & \multirow[b]{2}{*}{ Acceptor } & \multicolumn{2}{|c|}{ Occupancy (\%) } \\
\hline & & & Dark & Light \\
\hline 1 & 61GLN(E21) & 126FMC(N1) & - & 48,10 \\
\hline 2 & $126 \mathrm{FMC}(014)$ & 61GLN(NE2) & - & 71,60 \\
\hline 3 & 126FMN(O12) & 56ASN(OD1) & 70,70 & 84,00 \\
\hline 4 & 126FMN(H3) & 89ASN(OD1) & 89,30 & 91,40 \\
\hline 5 & 123VAL(HN) & 98TRP(O) & 74,70 & 66,70 \\
\hline 6 & 121VAL(HN & 100LEU(O) & 69,30 & 80,20 \\
\hline 7 & 121VAL(HN) & 99ASN(OD1) & 21,30 & - \\
\hline 8 & 120GLN(E21) & $126 \mathrm{FMN}(\mathrm{O} 4)$ & 62,70 & 65,40 \\
\hline *9 & 120GLN(E21) & 99ASN(OD1) & 40,00 & - \\
\hline 10 & $120 \mathrm{GLN}(\mathrm{HN})$ & 21THR(O) & 96,00 & 84,00 \\
\hline 11 & 119VAL(HN) & 102THR(O) & 96,00 & 93,80 \\
\hline 12 & 118GLY(HN) & 23VAL(O) & 96,00 & 100,00 \\
\hline 13 & 117VAL(HN) & 104THR(O) & 98,70 & 98,80 \\
\hline 14 & 116PHE(HN) & 25ALA(O) & 98,70 & 100,00 \\
\hline 15 & 115LYS(HN) & 106ILE(O) & 93,30 & 98,80 \\
\hline 16 & 114SER(HN) & 106ILE(O) & 93,30 & 87,70 \\
\hline 17 & 112ARG(H21) & 110ASP(OD2) & 56,00 & 42,00 \\
\hline 18 & 112ARG(H21) & 110ASP(OD1) & 45,30 & 63,00 \\
\hline 19 & 112ARG(HE) & 110ASP(OD2) & 42,70 & 61,70 \\
\hline 20 & 112ARG(HE) & 110ASP(OD1) & 50,70 & 43,20 \\
\hline 21 & $111 \mathrm{GLY}(\mathrm{HN})$ & 108THR(O) & 86,70 & 88,90 \\
\hline 22 & 108THR(HG1) & 112ARG(N) & 90,70 & 81,50 \\
\hline 23 & 108THR(HG1) & 110ASP(OD2) & 54,70 & 43,20 \\
\hline 24 & 108THR(HG1) & 110ASP(OD1) & 49,30 & 71,60 \\
\hline 25 & 108THR(HN) & 112ARG(O) & 98,70 & 96,30 \\
\hline 26 & 106ILE(HN) & 115LYS(O) & 98,70 & 100,00 \\
\hline 27 & 104THR(HN) & 117VAL(O) & 98,70 & 100,00 \\
\hline 28 & 103VAL(HN) & $83 \mathrm{CYS}(\mathrm{O})$ & 98,70 & 98,80 \\
\hline 29 & 102THR(HN) & 119VAL(O) & 94,70 & 97,50 \\
\hline 30 & 101LEU(HN) & 85VAL(O) & 96,00 & 97,50 \\
\hline 31 & 100LEU(HN) & 121VAL(O) & 80,00 & 84,00 \\
\hline 32 & 99ASN(D21) & 89ASN(OD1) & 81,30 & 74,10 \\
\hline 33 & $99 \mathrm{ASN}(\mathrm{HN})$ & 87LEU(O) & 98,70 & 98,80 \\
\hline 34 & 98TRP(HN) & 124THR(OG1) & 76,00 & 67,90 \\
\hline 35 & 97PHE(HN) & 89ASN $(0)$ & 100,00 & 100,00 \\
\hline 36 & 95THR(HG1) & 93ASP(OD2) & 66,70 & 48,10 \\
\hline 37 & 95THR(HG1) & 93ASP(OD1) & 46,70 & 61,70 \\
\hline 38 & 95THR(HN) & 93ASP(OD2) & 44,00 & 37,00 \\
\hline 39 & 95THR(HN) & 93ASP(OD1) & 26,70 & 50,60 \\
\hline 40 & $94 \mathrm{GLY}(\mathrm{HN})$ & 91ARG(O) & 77,30 & 76,50 \\
\hline 41 & 92LYS(HZ1) & 51GLU(OE2) & 54,70 & 61,70 \\
\hline 42 & 92LYS(HZ1) & 51GLU(OE1) & 64,00 & 64,20 \\
\hline 43 & 92LYS(HN) & 45THR(O) & 84,00 & 92,60 \\
\hline 44 & 91ARG(H21) & 93ASP(OD2) & 28,00 & - \\
\hline 45 & 91ARG(H21) & 93ASP(OD1) & 36,00 & 11,10 \\
\hline 46 & 91ARG(H11) & 95THR(OG1) & 34,70 & 59,30 \\
\hline 47 & 91ARG(HE) & 93ASP(OD2) & 32,00 & 13,60 \\
\hline 48 & 91ARG(HE) & 93ASP(OD1) & 29,30 & 14,80 \\
\hline 49 & 91ARG(HN) & 95THR(O) & 100,00 & 100,00 \\
\hline 50 & 90TYR(HN) & 6OLEU(O) & 92,00 & 96,30 \\
\hline 51 & 89ASN(D21) & $126 \mathrm{FMN}(\mathrm{O} 2)$ & 89,30 & 85,20 \\
\hline 52 & 89ASN(D21) & 88LEU(O) & 25,30 & 54,30 \\
\hline 53 & 89ASN(D21) & 6OLEU(O) & 45,30 & 18,50 \\
\hline 54 & 89ASN(HN) & 97PHE(O) & 100,00 & 98,80 \\
\hline 55 & 87LEU(HN) & 99ASN(O) & 98,70 & 93,80 \\
\hline 56 & 85VAL(HN) & 101LEU(O) & 94,70 & 100,00 \\
\hline 57 & 84SER(HG1) & 102THR(OG1) & 58,70 & 79,00 \\
\hline 58 & $83 \mathrm{CYS}(\mathrm{HN})$ & 103VAL(O) & 98,70 & 91,40 \\
\hline 59 & $81 \mathrm{GLU}(\mathrm{HN})$ & 76ALA(O) & 100,00 & 95,10 \\
\hline 60 & $80 \mathrm{GLY}(\mathrm{HN})$ & 76ALA(O) & 60,00 & 39,50 \\
\hline 61 & 79LYS(HZ1) & $75 \mathrm{ASP}(\mathrm{OD} 2)$ & 37,30 & 40,70 \\
\hline 62 & $79 \mathrm{LYS}(\mathrm{HZ1})$ & 75ASP(OD1) & 24,00 & 55,60 \\
\hline 63 & $79 L Y S(H N)$ & 75ASP(O) & 92,00 & 90,10 \\
\hline 64 & 78LYS(HN) & 74ARG(O) & 96,00 & 95,10 \\
\hline 65 & 77ILE(HN) & 73ILE(O) & 98,70 & 98,80 \\
\hline 66 & 76ALA(HN) & $72 L Y S(O)$ & 98,70 & 91,40 \\
\hline 67 & 75ASP(HN) & $71 \mathrm{GLN}(\mathrm{O})$ & 97,30 & 96,30 \\
\hline 68 & 74ARG(H21) & 126FMN(O3P) & 13,30 & 19,80 \\
\hline 69 & 74ARG(H21) & 126FMN(O2P) & 13,30 & 25,90 \\
\hline 70 & 74ARG(H11) & 126FMN(O2P) & 21,30 & 25,90 \\
\hline
\end{tabular}

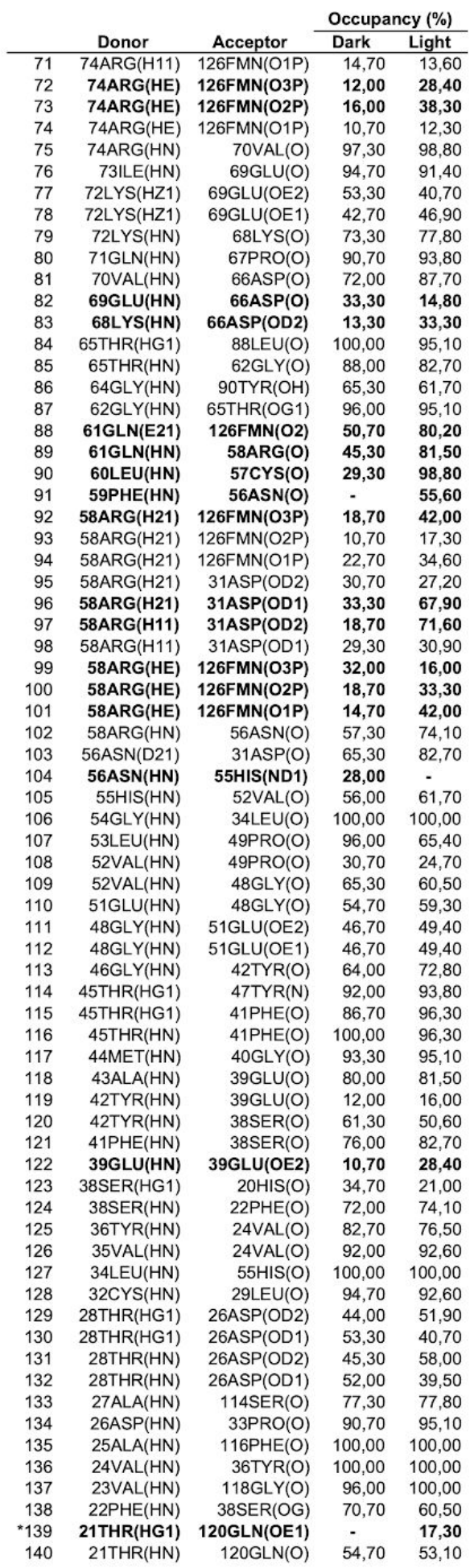

- Occupancy $\leqq 10 \%,{ }^{*}$ Hydrogen bond involving the conserved GLN120 
Table S2: Occupancy of three salt bridges potentially involved in signal transduction. A salt bridge is considered present in the best dark and light models if the side chains of the oppositely charged residues are within a radius of $6 \AA$.

\begin{tabular}{|c|cc|cc|}
\multicolumn{2}{c}{} & & \multicolumn{2}{c}{ Occupancy (\%) } \\
\cline { 4 - 5 } \multicolumn{2}{c}{ Salt Bridge } & Dark & Light \\
\hline 1 & ASP122 & ARG91 & 10,67 & 0,00 \\
2 & GLU51 & LYS92 & 98,67 & 100,00 \\
3 & GLU81 & LYS107 & 30,67 & 54,32 \\
\hline
\end{tabular}

\section{References}

1. Notredame, C., Higgins, D. G. \& Heringa, J. T-coffee: A novel method for fast and accurate multiple sequence alignment. J. Mol. Biol. 302, 205-217 (2000).

2. Robert, X. \& Gouet, P. Deciphering key features in protein structures with the new ENDscript server. Nucleic Acids Res. 42, (2014).

3. Šali, A. \& Blundell, T. L. Comparative protein modelling by satisfaction of spatial restraints. J. Mol. Biol. 234, 779-815 (1993). 